\title{
Role of Carbon Monoxide and Biliverdin in Renal Ischemia/Reperfusion Injury
}

\author{
G. Li Volti ${ }^{a} \quad$ L.F. Rodella $^{a}$ C. Di Giacomo ${ }^{b} \quad$ R. Rezzani ${ }^{a} \quad$ R. Bianchi ${ }^{a} \quad$ E. Borsani ${ }^{a}$ \\ D. Gazzoloc R. Motterlini ${ }^{d}$
}

a Division of Human Anatomy, Department of Biomedical Sciences and Biotechnology, University of Brescia, Brescia, ${ }^{b}$ Department of Biological Chemistry, Medicinal Chemistry and Molecular Biology, University of Catania, ${ }^{\mathrm{C}} \mathrm{G}$. Garibaldi Hospital, Catania, Italy; and dVascular Biology Unit, Department of Surgical Research, Northwick Park Institute for Medical Research, Harrow, UK

\section{Key Words}

Heme oxygenase $\cdot$ Bilirubin $\cdot$ Biliverdin $\cdot$ Carbon

monoxide $\cdot$ Kidney $\cdot$ Ischemia/reperfusion

\begin{abstract}
Heme oxygenase $(\mathrm{HO})$ isoforms catalyze the conversion of heme to carbon monoxide (CO) and biliverdin/bilirubin with a concurrent release of iron. There is strong evidence that $\mathrm{HO}$ activity and products play a major role in renoprotection, however the exact molecular mechanisms underlying the beneficial effects exerted by this pathway are not fully understood. This review is aimed at illustrating the possible mechanism/s by which $\mathrm{HO}$ is renoprotective in the context of ischemia/reperfusion. We will first analyze the effects of exogenous administration of bilirubin/biliverdin and $\mathrm{CO}$ and then describe their biological activities once generated endogenously following stimulation of the $\mathrm{HO}$ pathway by either pharmacological means or gene targeting-mediated approaches.

Copyright $\odot 2006$ S. Karger AG, Basel
\end{abstract}

Renal ischemia is a consequence of arterial occlusion, shock and organ transplantation and is a common cause of renal cell death, delayed graft function, renal graft re- jection and acute renal failure (ARF). The mortality rate of ARF remains between 50 and $70 \%$ among patients in intensive care who require dialysis, and ranges between 25 and $100 \%$ in postoperative patients suffering from ARF [1-3]. The prognosis is complicated by the fact that reperfusion, although essential for the survival of ischemic renal tissue, causes additional damage, contributing to the renal dysfunction and injury associated with ischemia/reperfusion injury (I/R) of the kidney [4]. Renal damage is triggered by a complex series of biochemical events which include among others oxidative stress and the release of pro-inflammatory mediators. Thus, the role of endogenous antioxidant and cytoprotective enzymes that can be engaged by the cellular system to counteract effectively the progression of renal pathological events assumes great importance. More specifically, and in the context of novel strategies for therapeutic applications, the modulation of heme oxygenases ( $\mathrm{HO}$ ) activity is emerging as a fascinating and promising target in renoprotection.

$\mathrm{HO}$ isoforms catalyze the conversion of heme to carbon monoxide (CO) and biliverdin/bilirubin with a concurrent release of iron, which can drive the synthesis of ferritin for iron sequestration $[5,6]$. HO is the limiting step in heme degradation and, consequently, plays a critical role in regulating the levels of cellular heme available

\section{KARGER \\ Fax +41613061234 E-Mail karger@karger.ch} www.karger.com (c) 2006 S. Karger AG, Basel

$1660-2129 / 06 / 1044-0135 \$ 23.50 / 0$

Accessible online at:

www.karger.com/nee
Dr. Giovanni Li Volti, MD, PhD

Piazza G. Sciuti, 29

IT-95131 Catania (Italy)

Tel. +39 095312 320, Fax +39095 312320

E-Maillivolti@unict.it 
for structural and functional heme-dependent proteins [7]. To date, two HO isoforms have been shown to be catalytically active in heme degradation, and each is encoded by a different gene $[6,8]$. HO-1, the inducible isoform, is found ubiquitously in all organs with the exception of some adult brain cell populations [9] and is rapidly and transiently expressed by a range of stressful stimuli; in contrast, HO-2 is the constitutive isozyme which, apart from controlling the basal levels of heme in the majority of cells, may also mediate important physiological effects such as vasodilatation, neurotransmission and oxygen sensing $[7,10]$.

The specific localization of $\mathrm{HO}$ isoforms within the kidney may have important implications with regard to renal vascular and tubular function. Under normal conditions, positive staining of $\mathrm{HO}-1$ protein is slightly detectable either in the cortex or in the outer medulla, whereas it has been shown to increase significantly within $3 \mathrm{~h}$ after reperfusion, to reach a maximum at $6 \mathrm{~h}$ and to decrease rapidly by $9 \mathrm{~h}$ reaching basal concentration by $12 \mathrm{~h}$ [11]. As far as HO-2 is concerned, it has been shown that this constitutive enzyme is expressed primarily in the arteriolar homogenates and to a lesser extent in the thick ascending limb (TAL) and proximal tubules [12]. So far, several studies have recognized the protective effects of HO-1 in different models of renal injury, however the precise mechanism by which increased HO-1 activity affords protection has yet to be proven definitively.

Bilirubin is a potent antioxidant tetrapyrrole that confers cellular protection against oxidative stress. Bilirubin itself is oxidized to biliverdin and then recycled by biliverdin reductase back to bilirubin, thus providing the cell with a redox couple of extraordinary and efficient antioxidant properties [13]. Recently, Adin et al. [14] demonstrated that bilirubin treatment resulted in a significant improvement of renal vascular resistance, urine output, glomerular filtration rate, tubular function and mitochondrial integrity after I/R injury and reported that beneficial effects on kidney viability were achieved most consistently with a dose of $10 \mu \mathrm{M}$ bilirubin. The authors also showed that although no significant improvement was observed on histological morphology following I/R, electron microscopy of the outer medullary stripe suggested that $10 \mu \mathrm{m}$ bilirubin treatment provided a specific protective effect at the level of the TAL demonstrating that mitochondrialintegrity (swelling and architecture of cristae) was better preserved in the bilirubin-treated kidneys. Consistent with these observations, Clark et al. [15] have previously reported that nanomolar concentrations of bilirubin protects myocardial tissue against I/R injury and that both contractile function and mitochondrial integrity were improved following treatment with the bile pigment. Demirogullari et al. [16] showed that, under their experimental conditions, bilirubin administration $(20 \mathrm{mg} / \mathrm{kg})$ at the time of reperfusion did not attenuate renal I-R injury; however, the use of hemin, a strong $\mathrm{HO}-1$ inducer, was able to ameliorate renal functionality and oxidative stress markers. Furthermore, Nakao et al. [17] showed that in renal transplant recipients, treatment with $\mathrm{CO}$ gas or biliverdin alone failed to recover creatinine clearance decrease as well as proteinuria; by contrast, all these parameters were normalized by the concomitant administration of CO gas and biliverdin. The same authors observed that rat treatment with biliverdin $2 \mathrm{~h}$ before renal transplantation resulted in a significant reduction of ED1+ macrophages infiltration and inflammatory mediators such as IL- 6 , IL-1 $1 \beta$, ICAM- 1 and iNOS. The effects of biliverdin on iNOS expression and activity is of great relevance; in fact, nitric oxide (NO) release seems to play a key role during renal I/R injury since numerous in vivo and in vitro investigations have demonstrated how inhibition of the expression or activity of iNOS, or absence of iNOS itself, can ameliorate or prevent renal I/R injury [18].

Recent studies have indicated that also CO inhalation therapy is able to protect renal grafts from ischemic damage in experimental models. In this regard, Neto et al. [19] showed that, in a rat model of transplant-induced I/ $\mathrm{R}$ injury, administration of $250 \mathrm{ppm}$ CO gas to the recipients resulted in a significant improvement of graft renal function as measured by glomerular filtration rate and creatinine levels. The authors also observed ultrastructural improvement following $\mathrm{CO}$ inhalation by using transmission electron microscopy evidencing viable podocytes, preservation of foot processes, less frequent vacuolization and maintenance of internal cellular architecture. Furthermore, $\mathrm{CO}$ inhalation resulted in a significant reduction of tubular epithelial cells apoptosis and, similarly to biliverdin administration, showed a significant decrease in IL-6, IL-1 $\beta$, ICAM-1, iNOS and nitrite/nitrate formation.

In line with these results and by using a $\mathrm{CO}$ carrier that delivers CO in biological systems, Vera et al. [20] showed that administration of the water-soluble $\mathrm{Ru}(\mathrm{CO})_{3} \mathrm{Cl}$ (glycinate) (CORM-3) $1 \mathrm{~h}$ before the onset of ischemia significantly decreased the levels of plasma creatinine $24 \mathrm{~h}$ after reperfusion as compared with vehicletreated mice. In addition, Sandouka et al. [21] demonstrated that kidneys flushed with and stored in cold Cel- 


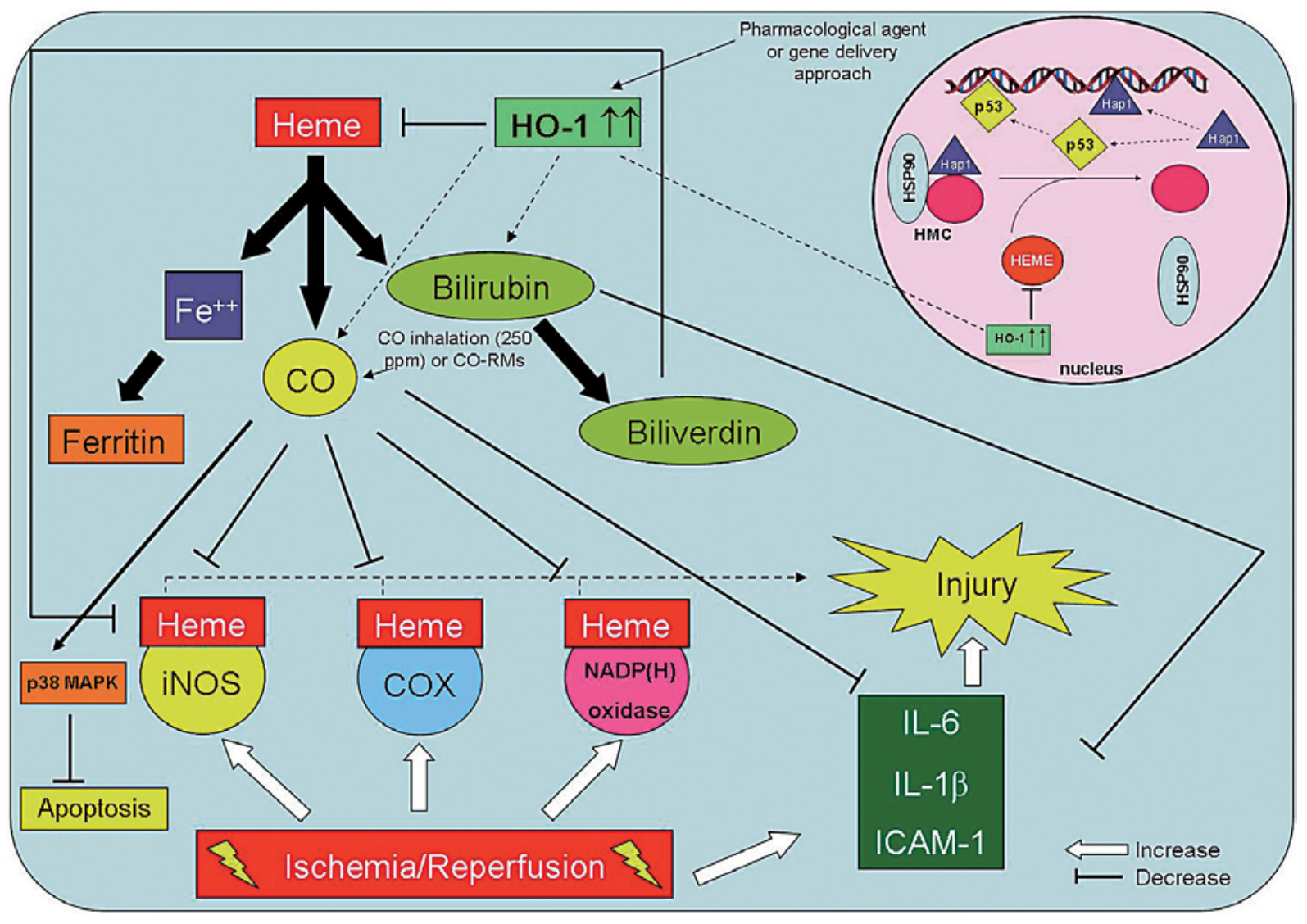

Fig. 1. Schematic representation of possible mechanisms involved in HO-mediated renoprotection.

sior solution supplemented with CORM-3 or CORM-A1, a boron-containing carbonate that releases $\mathrm{CO}$ at a slow rate, displayed at reperfusion a significantly higher perfusion flow rate, glomerular filtration rate, and sodium and glucose reabsorption rates compared to control kidneys flushed with Celsior solution alone. Interestingly, the same authors showed that the respiratory control index from kidney mitochondria treated with CO-RMs was markedly increased suggesting that CO may share with biliverdin some beneficial effects on the preservation of mitochondrial energy metabolism after ischemic events.

Taken together, the above-mentioned studies demonstrate that exogenous biliverdin/bilirubin and CO exert strong antioxidant and protective activities during renal I/R injury, probably by different mechanisms as suggested by the additive protection observed when the two compounds are administered simultaneously [17]. This hypothesis is further substantiated by studies where the use of a pharmacological agent leads to increased HO activity with a concomitant release of bilirubin/biliverdin and CO. Indeed, Toda et al. [11] showed that pretreatment with tin chloride, a potent and specific inducer of renal $\mathrm{HO}-1$, ameliorated the ischemic renal injury as judged by the significant decrease in serum creatinine and blood urea nitrogen levels and reduction in tubular epithelial cell injury. Interestingly, the same authors showed that pharmacological induction of HO-1 leads also to a significant decrease in microsomal heme content which may represent a further cytoprotective mechanism; in fact, heme functions as prosthetic group of important enzymes playing a key role in the pathophysiological processes of renal injury such as NOS, cyclooxygenase (COX) and NADP(H) oxidase. Similarly, Li Volti et al. [22] reported that pharmacological or retroviral-mediated induction of HO-1 leads to heme depletion and consequently to a decreased COX activity. Furthermore, Taille et al. [23] elegantly demonstrated that a decrease in heme content due to HO-1 activation limits heme availability for maturation of the gp91(phox) subunit and assembly of the functional $\mathrm{NAD}(\mathrm{P}) \mathrm{H}$ oxidase and thus attenuating 
free radicals formation and oxidative stress damage. In order to avoid this inconvenient, Quan et al. [24] used a retroviral-mediated approach for $\mathrm{HO}-1$ induction in the kidney by using an adenoviral vector expressing the human HO-1 under the control of the TALH-specific promoter $\mathrm{Na}^{+}-\mathrm{K}^{+}-\mathrm{Cl}^{-}$cotransporter (NKCC2 promoter) and demonstrated a site-specific expression of HO-1 and a TALH-selective protection versus oxidative stress.

The molecular mechanisms underlying the anti-inflammatory and antiapoptotic effects of $\mathrm{CO}$ remain to be fully elucidated. However, a number of studies have shown the possible involvement of the MAPK pathways and in particular of p38 MAPK activation by CO in preventing inflammatory and apoptotic processes. In this regard, Otterbein et al. [25] reported that in the presence of $250 \mathrm{ppm}$ CO, LPS-induced activation of the MAPK ERK1/ERK2 and JNK was not affected, however p38 MAPK activation was significantly increased. Furthermore, Zhang et al. [26] demonstrated that in an anoxiareoxygenation experimental model, $\mathrm{CO}$ exerts its antiapoptotic effects via stimulation of the p38 MAPK pathway.

Several other mechanisms of protection have been advocated to explain $\mathrm{HO}-1$-mediated antioxidant effects in the kidney including the cooperation by ferritin [27] and the possibility that, under certain oxidative stress conditions [28], HO-1 could translocate into the nucleus where it may act as a regulator of some heme-dependent transcriptional factors or facilitate the shuttling of other important molecules. Irrespective of the precise mechanism(s) involved, we have learned that the products of HO-1 can independently or synergistically afford endogenous protection to renal cells and contribute to preserve kidney function after I/R injury and transplantation (fig. 1). It is important to note that the only known human case of HO-1 deficiency was affected by persistent proteinuria and hematuria with biochemical evidence of renal tubular injury, in addition to severe oxidative stress inflicted to the vascular endothelium [29].

Taken together, the above-mentioned results suggest that the HO-1 pathway serves as an obligatory inducible sentinel of the kidney function and both $\mathrm{CO}$ and biliverdin can be used in exploring effective therapeutic avenues to maximize renoprotection in the treatment of renal diseases. By contrast, despite these promising findings, it is perceived that a therapy based on $\mathrm{CO}$ inhalation presents several disadvantages since this gaseous molecule is not easy to control, it is difficult to store, and it leads to decreased oxygen-carrying capacity of blood and to undesired effects after prolonged exposure. This problematic aspect has been solved in part by an alternative and promising approach that is the use of CO-RMs, which have been shown to exert vascular and cytoprotective activities that are reminiscent of the HO-1/CO pathway [30]; however, further studies are necessary to optimize CORMs and chemically engineer new compounds that may possess all the necessary characteristics to provide a powerful tool in the organ preservation strategies. As far as the pharmacological induction of HO-1 is concerned, this approach may also be limiting since specific site expression along the nephron and a controlled amount of by-products being formed after stimulation of HO-1 are not easy to achieve. In this regard, exogenous administration of HO-1 by gene transfer could be a more specific and attractive approach; however, the use of viral vector in the clinical practice is currently very limited and more studies are necessary to show the efficacy and safety of selectively modulating HO-1 induction by the use of specific promoters.

\section{References}

1 Spiegel DM, Ullian ME, Zerbe GO, Berl T: Determinants of survival and recovery in acute renal failure patients dialyzed in intensive-care units. Am J Nephrol 1991;11:4447.

-2 Novis BK, Roizen MF, Aronson S, Thisted RA: Association of preoperative risk factors with postoperative acute renal failure. Anesth Analg 1994;78:143-149.

\footnotetext{
-3 Zanardo G, Michielon P, Paccagnella A, Rosi $\mathrm{P}$, Calo M, Salandin V, Da Ros A, Michieletto F, Simini G: Acute renal failure in the patient undergoing cardiac operation. Prevalence, mortality rate, and main risk factors. J Thorac Cardiovasc Surg 1994;107:1489-1495.

4 Paller MS: The cell biology of reperfusion injury in the kidney. J Investig Med 1994;42: 632-639.

-5 Eisenstein RS, Garcia-Mayol D, Pettingell W, Munro HN: Regulation of ferritin and heme oxygenase synthesis in rat fibroblasts by different forms of iron. Proc Natl Acad Sci USA 1991;88:688-692.
}

\footnotetext{
6 Shibahara S, Yoshizawa M, Suzuki H, Takeda K, Meguro K, Endo K: Functional analysis of cDNAs for two types of human heme oxygenase and evidence for their separate regulation. J Biochem (Tokyo) 1993;113:214218.

7 Abraham NG, Lin JH, Schwartzman ML, Levere RD, Shibahara S: The physiological significance of heme oxygenase. Int J Biochem 1988;20:543-558.
} 
8 McCoubrey WK Jr, Ewing JF, Maines MD: Human heme oxygenase-2: characterization and expression of a full-length cDNA and evidence suggesting that the two HO-2 transcripts may differ by choice of polyadenylation signal. Arch Biochem Biophys 1992; 295:13-20.

-9 Ewing JF, Haber SN, Maines MD: Normal and heat-induced patterns of expression of heme oxygenase-1 (HSP32) in rat brain: hyperthermia causes rapid induction of mRNA and protein. J Neurochem 1992;58:11401149.

-10 Zhang Y, Furuyama K, Adachi T, Ishikawa K, Matsumoto H, Masuda T, Ogawa K, Takeda K, Yoshizawa M, Ogawa H, Maruyama Y, Hida W, Shibahara S: Hypoxemia and attenuated hypoxic ventilatory responses in mice lacking heme oxygenase-2: evidence for a novel role of heme oxygenase-2 as an oxygen sensor. Adv Exp Med Biol 2006;580:161166.

-11 Toda N, Takahashi T, Mizobuchi S, Fujii H, Nakahira K, Takahashi S, Yamashita M, Morita K, Hirakawa M, Akagi R: Tin chloride pretreatment prevents renal injury in rats with ischemic acute renal failure. Crit Care Med 2002;30:1512-1522.

-12 Da Silva JL, Zand BA, Yang LM, Sabaawy HE, Lianos E, Abraham NG: Heme oxygenase isoform-specific expression and distribution in the rat kidney. Kidney Int 2001;59: 1448-1457.

- 13 Stocker R, Yamamoto Y, McDonagh AF, Glazer AN, Ames BN: Bilirubin is an antioxidant of possible physiological importance. Science 1987;235:1043-1046.

$\checkmark 14$ Adin CA, Croker BP, Agarwal A: Protective effects of exogenous bilirubin on ischemiareperfusion injury in the isolated, perfused rat kidney. Am J Physiol Renal Physiol 2005; 288:F778-F784.
15 Clark JE, Foresti R, Sarathchandra P, Kaur H, Green CJ, Motterlini R: Heme oxygenase1-derived bilirubin ameliorates postischemic myocardial dysfunction. Am J Physiol Heart Circ Physiol 2000;278:H643-H651.

-16 Demirogullari B, Ekingen G, Guz G, Bukan N, Erdem O, Ozen IO, Memis L, Sert S: A comparative study of the effects of hemin and bilirubin on bilateral renal ischemia reperfusion injury. Nephron Exp Nephrol 2005;103:e1-e5.

17 Nakao A, Neto JS, Kanno S, Stolz DB, Kimizuka K, Liu F, Bach FH, Billiar TR, Choi AM, Otterbein LE, Murase N: Protection against ischemia/reperfusion injury in cardiac and renal transplantation with carbon monoxide, biliverdin and both. Am J Transplant 2005;5:282-291.

18 Goligorsky MS, Brodsky SV, Noiri E: Nitric oxide in acute renal failure: NOS versus NOS. Kidney Int 2002;61:855-861.

19 Neto JS, Nakao A, Kimizuka K, Romanosky AJ, Stolz DB, Uchiyama T, Nalesnik MA, Otterbein LE, Murase N: Protection of transplant-induced renal ischemia-reperfusion injury with carbon monoxide. Am J Physiol Renal Physiol 2004;287:F979-F989.

20 Vera T, Henegar JR, Drummond HA, Rimoldi JM, Stec DE: Protective effect of carbon monoxide-releasing compounds in ischemia-induced acute renal failure. J Am Soc Nephrol 2005;16:950-958.

-21 Sandouka A, Fuller BJ, Mann BE, Green CJ, Foresti R, Motterlini R: Treatment with CORMs during cold storage improves renal function at reperfusion. Kidney Int 2006;69: 239-247.

22 Li Volti G, Seta F, Schwartzman ML, Nasjletti A, Abraham NG: Heme oxygenase attenuates angiotensin II-mediated increase in cyclooxygenase- 2 activity in human femoral endothelial cells. Hypertension 2003;41: 715-719.

$\checkmark 23$ Taille C, El Benna J, Lanone S, Dang MC, Ogier-Denis E, Aubier M, Boczkowski J: Induction of heme oxygenase-1 inhibits NAD (P)H oxidase activity by down-regulating cytochrome b558 expression via the reduction of heme availability. J Biol Chem 2004;279:28681-28688.
24 Quan S, Yang L, Shnouda S, Schwartzman ML, Nasjletti A, Goodman AI, Abraham NG: Expression of human heme oxygenase-1 in the thick ascending limb attenuates angiotensin II-mediated increase in oxidative injury. Kidney Int 2004;65:1628-1639.

25 Otterbein LE, Bach FH, Alam J, Soares M, Tao Lu H, Wysk M, Davis RJ, Flavell RA, Choi AM: Carbon monoxide has anti-inflammatory effects involving the mitogenactivated protein kinase pathway. Nat Med 2000;6:422-428.

26 Zhang X, Shan P, Otterbein LE, Alam J, Flavell RA, Davis RJ, Choi AM, Lee PJ: Carbon monoxide inhibition of apoptosis during ischemia-reperfusion lung injury is dependent on the p38 mitogen-activated protein kinase pathway and involves caspase 3 . J Biol Chem 2003;278:1248-1258.

27 Balla G, Jacob HS, Balla J, Rosenberg M, Nath K, Apple F, Eaton JW, Vercellotti GM: Ferritin: a cytoprotective antioxidant strategem of endothelium. J Biol Chem 1992;267: 18148-18153.

28 Li Volti G, Ientile R, Abraham NG, Vanella A, Cannavo G, Mazza F, Curro M, Raciti G, Avola R, Campisi A: Immunocytochemical localization and expression of heme oxygenase- 1 in primary astroglial cell cultures during differentiation: effect of glutamate. Biochem Biophys Res Commun 2004;315: 517-524.

29 Ohta K, Yachie A, Fujimoto K, Kaneda H, Wada T, Toma T, Seno A, Kasahara Y, Yokoyama H, Seki H, Koizumi S: Tubular injury as a cardinal pathologic feature in human heme oxygenase-1 deficiency. Am J Kidney Dis 2000;35:863-870.

30 Motterlini R, Mann BE, Foresti R: Therapeutic applications of carbon monoxide-releasing molecules. Expert Opin Investig Drugs 2005;14:1305-1318. 